\title{
Les approches de précaution dans le secteur alimentaire
}

\author{
Pierre-Etienne Bouillot ${ }^{1}$
}

Résumé :

De la production à la transformation, le secteur alimentaire fait face à des risques incertains pour la santé qui impliquent la mobilisation d'approches de précaution.

Dans le règlement $(\mathrm{CE}) \mathrm{n}^{\circ}$ 178/2002 établissant les principes généraux de la législation alimentaire, le principe de précaution a été énoncé précisément. Sans surprise, il implique de prendre des mesures de gestion en cas de suspicion d'un risque. Il s'adresse aux pouvoirs publics. La jurisprudence est venue préciser les critères de sa mise en œuvre en marquant une relative dépendance du gestionnaire des risques à l'avis de l'évaluateur, mais les mesures de gestion de risques sont parfois légistiquement discutables et cacophoniques. Dans un contexte de désengagement de l'État, les exploitants du secteur alimentaire sont également concernés par la précaution, mais le contour de leurs obligations dans ce domaine reste encore imprécis. À défaut d'interprétation jurisprudentielle, davantage de transparence pourrait venir au support d'une meilleure gestion du risque incertain.

Mots clés : précaution, alimentation, risque, incertitude, transparence

Le principe de précaution a déjà été abondamment discuté dans la doctrine et sa dimension alimentaire n'y a pas échappé ${ }^{2}$. Pourtant, il reste trop souvent incompris et accusé notamment de porter atteinte à l'innovation et à la compétitivité ${ }^{3}$. Pourtant, selon la Commission européenne, les effets indésirables de ce principe n'ont pas de fondement, au moins dans le secteur alimentaire ${ }^{4}$.

Dans ce secteur, le principe de précaution apparaît implicitement dans la réglementation et la jurisprudence européenne dès la fin des années 1980. Le domaine de l'alimentation est original à plusieurs titres, car il se situe au carrefour d'enjeux économiques, sanitaires et environnementaux, mais aussi culturels. À la différence du médicament, l'aliment bénéficie d'une présomption d'innocuité. Par principe, sa mise sur le marché et sa circulation sont donc libres et c'est principalement en cas de risque sanitaire pour le consommateur que celles-ci sont atténuées, que le risque soit avéré, ou suspecté ${ }^{5}$.

Les leçons du passé, en particulier de la crise de la vache folle, ont amené l'Union européenne à légiférer spécialement pour ce secteur. Le principe de précaution a été énoncé plus

\footnotetext{
${ }^{1}$ Maître de conférences à AgroParisTech (Université Paris-Saclay), chercheur à l'Institut de Recherche Juridique de la Sorbonne. L'auteur peut être contacté à l'adresse pierre-etienne.bouillot @agroparistech.fr.

${ }^{2}$ F. Collart Dutilleul, «Le principe de précaution dans le règlement communautaire du 28 janvier 2002 », in A. Massart, Ed. Giuffre (dir.), Prodotti agricoli e sicurezza alimentare, 2003, p. 239

${ }^{3}$ V. par ex. : Assemblée Nationale, Proposition de loi constitutionnelle visant à retirer le principe de précaution du bloc de constitutionnalité, $\mathrm{n}^{\circ}$ 2033, 13 juin 2014 ; R.-M. Borges, « Le statut des nouvelles techniques de sélection des plantes : entre incohérences juridiques et démission politique », RD rur., $\mathrm{n}^{\circ} 471$, mars 2019, ét. 8.

${ }^{4}$ European Commission, «The Regulatory Fitness and Performance Programme Evaluation of the General Food Law (Regulation (EC) No 178/2002), SWD(2018) 38 final », January 2018, p. 39.

${ }^{5}$ Les notions de risque et de danger sont définies par le règlement (CE) $\mathrm{n}^{\circ}$ 178/2002. Le risque est « une fonction de la probabilité et de la gravité d'un effet néfaste sur la santé, du fait de la présence d'un danger » (Règlement CE n ${ }^{\circ}$ 178/2002, art. 3 9)). Ainsi, le danger est une composante du risque. Il est défini comme «un agent biologique, chimique ou physique présent dans les denrées alimentaires ou les aliments pour animaux, ou un état de ces denrées alimentaires ou aliments pour animaux, pouvant avoir un effet néfaste sur la santé » (Règlement CE n ${ }^{\circ}$ 178/2002, art. 3 14).)
} 
précisément dans le règlement $\mathrm{CE} \mathrm{n}^{\circ}$ 178/2002 établissant les principes généraux et les prescriptions générales de la législation alimentaire :

Son article 7 énonce notamment :

1. Dans des cas particuliers où une évaluation des informations disponibles révèle la possibilité d'effets nocifs sur la santé, mais où il subsiste une incertitude scientifique, des mesures provisoires de gestion du risque, nécessaires pour assurer le niveau élevé de protection de la santé choisi par la Communauté, peuvent être adoptées dans l'attente d'autres informations scientifiques en vue d'une évaluation plus complète du risque.

2. Les mesures adoptées en application du paragraphe 1 sont proportionnées et n'imposent pas plus de restrictions au commerce qu'il n'est nécessaire pour obtenir le niveau élevé de protection de la santé choisi par la Communauté, en tenant compte des possibilités techniques et économiques et des autres facteurs jugés légitimes en fonction des circonstances en question. Ces mesures sont réexaminées dans un délai raisonnable, en fonction de la nature du risque identifié pour la vie ou la santé et du type d'informations scientifiques nécessaires pour lever l'incertitude scientifique et réaliser une évaluation plus complète du risque.

Les critères classiques de la précaution y sont donc énoncés : un risque incertain, des mesures provisoires et proportionnées et un réexamen régulier de la mesure. À la différence de la définition prévue par la Charte de l'environnement par exemple, il n'est pas question ici de dommages graves et irréversibles, mais d'effets nocifs pour la santé. Le législateur européen s'en tient là. Nulle mention d'un seuil pour le déclenchement ni de la nature des mesures à adopter qui peuvent donc être très variées. C'est la nature même d'un principe : " une normeguide, abstraite et de portée générale, qui, pour assurer le respect d'une "valeur" socialement admise, vise à orienter des décisions et des comportements ainsi qu'à les apprécier ${ }^{6}$. C'est pourquoi le principe de précaution n'appelle pas un modèle d'action prédéfini. Les approches de précaution sont plurielles et ont d'ailleurs une normativité variable ${ }^{7}$. Dès lors, il est possible de considérer qu'il procède parfois plus du standard que du principe ${ }^{8}$. "Sans doute ce qui dérange, peut-être [encore], les juristes aujourd'hui, c'est que les standards ne doivent plus permettre le jugement de situations statiques, figées dans le temps, mais des situations évolutives ${ }^{9}$. Les juristes ne sont pas les seuls à être perturbés. Les exploitants, comme les consommateurs d'ailleurs, ont souvent besoin de certitudes : a-t-on le droit de mettre ce produit sur le marché ? D'utiliser tel additif ? Cette denrée alimentaire présente-t-elle un danger ? Quel risque encoure-t-on à la consommer ? C'est bien là toute la potentialité du principe de précaution : agir de manière provisoire face à l'incertain.

Sollicité dans le cadre du principe de l'analyse des risques, le principe de précaution permet aux gestionnaires du risque, c'est-à-dire aux pouvoirs publics, de choisir les mesures de prudence et de contrôle appropriées. Plus original, les exploitants du secteur alimentaire sont également concernés par les approches de précaution. Depuis l'entrée en vigueur du règlement (CE) $\mathrm{n}^{\circ} 178 / 2002$, les exploitants du secteur alimentaire, qu'ils soient producteurs, transformateurs ou distributeurs, ont également vu leurs obligations renforcées en matière de sécurité sanitaire des denrées alimentaires ${ }^{10}$, tant dans le domaine de la prévention des risques que dans la mise en place de mesures de précaution. Il s'agit en particulier d'une obligation de

\footnotetext{
${ }^{6}$ F. Collart Dutilleul, op. cit..

${ }^{7}$ N. De Sadeleer, Sécurité alimentaire et précaution, Sécurité alimentaire. Nouveaux enjeux et perspectives, Bruylant, 2013.

${ }^{8}$ D'où la qualification plus globale d'approche dans cette contribution

${ }^{9}$ L. Boy, « La nature juridique du principe de précaution », Nature Sciences Sociétés, 7, 1999.

${ }^{10}$ V. not. : J. Bombardier, «Fabrication et distribution d'un produit : de la mise sur le marché à la gestion des risques, quelles obligations et sanctions pour les professionnels ? », Revue de droit rural, n ${ }^{\circ} 68$, déc. 2018, étude 24.
} 
mise sur le marché de denrées alimentaires sûres et non dangereuses, d'une obligation de traçabilité, de la mise en place de mesures de retrait ou de rappel des produits en cas de risque avéré ou suspecté et encore d'une obligation d'information du consommateur. Même si le terme de précaution n'est pas expressément utilisé, la législation alimentaire générale prolonge donc l'obligation de prudence des entreprises jusqu'au risque suspecté ${ }^{11}$.

Sans faire le bilan des effets de ce principe dans le secteur alimentaire alors que cela fait une quinzaine d'années que ce règlement est entrée en vigueur, cette contribution propose davantage de développer un regard critique des approches de précaution mises en œuvre par les pouvoirs publics et les exploitants du secteur alimentaire. Les premières montrent la complexité de l'action publique dans un contexte sanitaire évolutif. Les pouvoirs publics tâtonnent et usent avec maladresse des outils juridiques qu'ils possèdent (I). À l'inverse, les exploitants semblent profiter de ces tâtonnements qui offrent un flou, certes insécurisant, mais propice à une certaine latence dans la prise de décisions restrictives de la libre-circulation des denrées alimentaires (II). Ce sont évidemment les deux faces d'une même pièce, car ces approches de précaution distinctes visent, de manière complémentaire, à éviter la paralysie du système face aux risques incertains. Plus largement, l'étude de ces approches témoigne aussi des faiblesses du système actuel. Si le système juridique appliqué à l'alimentation n'a jamais été aussi précis et structuré et, comme certains l'affirment, si une maîtrise croissante et substantielle des risques est mise en pratique, les consommateurs témoignent pourtant d'une certaine défiance à l'égard de maillons du secteur alimentaire et plus largement du système de l'analyse des risques ${ }^{12}$.

\section{Tâtonnements dans l'adoption de mesures de précaution par les pouvoirs publics}

Dans le bilan qualité de la législation alimentaire générale, la Commission précise que le principe de précaution a été invoqué par l'Union européenne dans un nombre limité de cas ${ }^{13}$. Disons-le d'emblée, au regard de la production normative dans le domaine des risques incertain, les pouvoirs publics apparaissent maladroits. L'usage d'actes juridiques inadaptés aux risques incertains (A) participe à la cacophonie de la communication sur les risques (B).

\section{A. Mésusages des actes juridiques concernant les risques suspectés}

Dans sa version publique, le principe de précaution a été interprété par la jurisprudence européenne dans le domaine alimentaire. La Cour de justice de l'Union européenne est venue notamment préciser que des mesures de précaution ne sauraient être justifiées par une approche purement hypothétique du risque. Ainsi, l'évaluation scientifique joue un rôle crucial en venant étayer le choix de la mesure adéquate par le gestionnaire du risque ${ }^{14}$. Lorsque la connaissance scientifique tâtonne ou que la controverse scientifique est vivace concernant un risque sanitaire lié à l'alimentation, le processus d'analyse des risques est dynamique. En tant que gestionnaires du risque, les pouvoirs publics (institutions européennes, États membres ou encore collectivités territoriales ...) doivent être réactifs pour adopter les mesures nécessaires pour assurer un niveau élevé de protection de la santé. Tant qu'elles sont proportionnées et, en particulier, qu'elles n'imposent pas plus de restrictions que nécessaire à la libre-circulation des marchandises, des mesures préventives peuvent être prises, même à très brève échéance comme

\footnotetext{
${ }^{11}$ Voy not. : Règlement CE n ${ }^{\circ}$ 178/2002, art. 19.

${ }^{12}$ Conseil National de l'Alimentation, Gestion de crise et communication, Enseignements tirés de la crise de l'ESB, avis $n^{\circ} 79,2017$.

${ }^{13}$ European Commission, « The Regulatory Fitness and Performance Programme Evaluation of the General Food Law (Regulation (EC) No 178/2002), SWD(2018) 38 final », publié le 15 janvier 2018, p. 39.

${ }^{14}$ Cette vision formelle peut être discutée du point de vue sociologique : D. Demortain, « Une société (de l'analyse) du risque? », Nat. Sci. Soc., 27, 2019.
} 
le confirme la jurisprudence de l'Union européenne ${ }^{15}$. Si, assez logiquement, une nouvelle publication scientifique est susceptible de rendre la norme juridique de gestion du risque obsolète, la Cour de justice a également admis que « des évaluations divergentes de ces risques peuvent légitimement être effectuées, sans nécessairement être fondées sur des données scientifiques différentes ou nouvelles $\gg{ }^{16}$.

Ces situations imposent une relative réactivité de la norme juridique en rapport avec les évolutions de la connaissance scientifique.

Il est alors plus adapté d'attribuer ce rôle au pouvoir exécutif qu'au pouvoir législatif pour remplir cette mission pour au moins deux raisons : l'une tient au temps d'édiction de la norme et l'autre à la compétence requise pour se saisir des faits scientifiques.

Par exemple, la voie législative a été utilisée pour suspendre de la mise sur le marché du bisphénol A (une molécule pouvant servir pour la fabrication de matériaux en contact avec les denrées alimentaires comme les emballages) ${ }^{17}$ comme pour celle du dioxyde de titane (un additif alimentaire blanchisseur et opacifiant utilisé notamment pour les pâtisseries) ${ }^{18}$.

Dans des circonstances d'incertitude, l'usage d'actes réglementaire paraît plus approprié. D'une part, parce qu'une mesure de précaution est par nature provisoire. Les procédures d'adoption des décrets et des arrêtés sont normalement plus brèves que celles des lois. De plus, il est plus simple d'abroger ou de modifier un acte réglementaire qu'un acte législatif pour les faire évoluer en fonction des nouvelles données scientifiques. D'autre part, du fait des objets techniques auxquels s'intéressent ces normes. Rappelons que le bisphénol A n'est pas le seul bisphénol pouvant entrer dans la composition des matériaux en contact avec les denrées alimentaires ${ }^{19}$ et que le dioxyde de titane est loin d'être le seul additif dont le risque pour la santé est incertain. Est-il raisonnable de confier une tâche qui pourrait être potentiellement répétitive et rapidement obsolète au législateur? Si les listes sont souvent utilisées en droit européen ${ }^{20}$, leurs mises à jour sont bien souvent issues de règlements d'exécution de la Commission et non de textes du colégislateur européen ${ }^{21}$.

Ce décalage entre le temps de la science et le temps du droit apparaît d'autant plus problématique lorsqu'il est couplé à des choix légistiques discutables. Si ces dispositions ont été validées par le Conseil constitutionnel ${ }^{22}$, tout du moins partiellement pour le bisphénol $\mathrm{A}^{23}$, il n'en demeure pas moins que ce choix a de quoi étonner. Ces mesures se limitent à un objet

\footnotetext{
15 TPI, 11 septembre 2002, Pfizer, T-13/99, Rec. 2002 p. II-03305, pt. 160.

${ }^{16}$ CJCE, 2 mars 2003, Danemark c. Commission, C-3/00, Rec. 2003, p. I-2643, pt. 63.

${ }^{17}$ La loi n $^{\circ} 2010-729$ du 30 juin 2010 modifiée par la loi n²012-1442 du 24 décembre 2012 suspend l'importation et la mise sur le marché à titre gratuit ou onéreux de tout conditionnement, contenant ou ustensile comportant du bisphénol $\mathrm{A}$ et destiné à entrer en contact direct avec toutes les denrées alimentaires à partir du 1er janvier 2015.

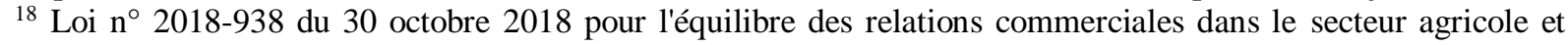
alimentaire et une alimentation saine, durable et accessible à tous, JORF n ${ }^{\circ} 0253 \mathrm{du} 1$ novembre 2018, art. 53.

${ }^{19}$ En lisant l'avis de l'Anses concernant les alternatives au Bisphénol A, on comprend que les substituts ne sont pas forcément moins toxiques, mais que l'on en sait moins sur leur toxicité. ANSES, Avis de l'Agence nationale de sécurité sanitaire de l'alimentation, de l'environnement et du travail relatif à l'évaluation des risques liés au Bisphénol A (BPA) pour la santé humaine et aux données toxicologiques et d'usage des bisphénols S, F, M, B, AP, AF, et BADGE, mars 2013.

${ }^{20}$ A titre d'exemple dans le domaine alimentaire : la liste des allégations nutritionnelles autorisées par le règlement $(\mathrm{CE}) \mathrm{n}^{\circ}$ 1924/2006, celle des additifs dans le règlement (CE) $\mathrm{n}^{\circ}$ 1333/2008 ou encore celle des aliments nouveaux prévue par le règlement (UE) $n^{\circ} 2015 / 2233$.

${ }^{21}$ V. par exemple Comm. UE, dir. 2011/8 du 28 janvier 2011modifiant la directive 2002/72/CE en ce qui concerne la restriction de l'utilisation du bisphénol A dans les biberons en plastique pour nourrissons: JOUE $n^{\circ}$ L $^{26}, 29$ janv. 2011, p. 11. Ce règlement fait explicitement référence au principe de précaution pour fonder la suspension de l'utilisation du BPA dans la fabrication et la mise sur le marché de biberons en polycarbonate pour nourrissons, «jusqu'à ce que l'on dispose de données scientifiques complémentaires permettant de clarifier la pertinence toxicologique de certains effets observés du BPA »

${ }^{22}$ Cons. const., 25 oct. 2018, $\mathrm{n}^{\circ} 2018-771$.

${ }^{23}$ Cons. const., 17 sept. 2015, n ${ }^{\circ} 2015-480$.
} 
très précis, alors que la loi se veut l'instrument de l'édiction de normes à portée générale. À regarder l'exposé des motifs de l'arrêté de suspension du dioxyde de titane ${ }^{24}$, les pouvoirs publics ont pris la mesure de l'importance de fonder scientifiquement et précisément leurs mesures de gestion de risque pour justifier une atteinte au principe de libre-circulation des marchandises et à certains intérêts économiques. L'entremise du législateur n'était pas nécessaire vu la référence à l'article L. 521-17 du Code de la consommation. En effet, par cet article, le législateur confiait déjà justement l'adoption de telles mesures au pouvoir exécutif. Cela a sans doute compliqué l'adoption de la mesure de suspension en la conditionnant à une situation d'urgence en présence d'un danger grave et immédiat.

Sans remettre en cause l'importance de l'apparence des aliments, le cas du dioxyde de titane met en évidence qu'entre un additif à vocation esthétique et un doute relatif à la sécurité sanitaire du consommateur, le choix n'a pas l'air si évident pour les pouvoirs publics, notamment dans un contexte de libre-circulation des marchandises. Cette dépendance des pouvoirs publics à la science n'est pourtant pas imposée par les textes, puisque «d'autres facteurs légitimes » peuvent être mobilisés pour choisir la mesure la plus appropriée ${ }^{25}$. Comme l'a souligné la Commission dans son bilan de qualité de la législation alimentaire générale, il est d'ailleurs mal aisé de déterminer si les mesures sont motivées par l'application du principe de précaution ou par la prise en compte d'autres facteurs légitimes ${ }^{26}$.

Si les acteurs ne restent pas dans le rôle qui leur est attribué par la législation, n'est-ce pas la confiance dans le système normatif qui s'affaiblit? Sans doute, le législateur souhaite-t-il montrer qu'il se préoccupe de la santé des consommateurs, mais dans ce cas il communique plus sur le risque qu'il ne le gère. Il fait un mésusage de l'outil législatif et entretien ce que certains appellent la cacophonie de la communication sur les risques sanitaires. Il est vrai que concernant la communication sur les risques, les droits et obligations des acteurs de l'analyse des risques sont moins clairement délimités.

\section{B. Un besoin de clarification de la communication sur les risques}

Ce volet de l'analyse des risques est défini à l'article 3 du règlement CE no 178/2002 comme « l'échange interactif, tout au long du processus d'analyse des risques, d'informations et d'avis sur les dangers et les risques, les facteurs liés aux risques et les perceptions des risques, entre les responsables de l'évaluation des risques et de la gestion des risques, les consommateurs, les entreprises du secteur alimentaire et du secteur de l'alimentation animale, les milieux universitaires et les autres parties intéressées, et notamment l'explication des résultats de l'évaluation des risques et des fondements des décisions prises en matière de gestion des risques $\gg^{27}$.

La nature de la communication caractérise le changement de paradigme opéré dans le cadre de la nouvelle législation européenne, en particulier concernant l'évolution du rôle des autorités administratives. Les relations entre l'administration et ses administrés étaient jusqu'alors surtout envisagées verticalement : l'administration était responsable de la sécurité sanitaire et encadrait les activités du secteur alimentaire en ce sens. L'information «descendait »de l'administration vers les opérateurs du secteur (transformateurs, distributeurs, transporteurs, etc.). Désormais, ces relations sont plus horizontales. Ainsi, la communication sur les risques

\footnotetext{
${ }^{24}$ Arrêté du 17 avril 2019 portant suspension de la mise sur le marché des denrées contenant l'additif E 171 (dioxyde de titane - TiO2), JORF n ${ }^{\circ} 097$ du 25 avril 2019. A ce propos, v. : S. Lacour, « Nanotechnologies. « Nanomatériaux dans l'alimentation. Vous reprendrez bien un peu de dioxyde de titane? », Cahiers Droit, Sciences \& Technologies, 2019.

${ }^{25}$ Articles $3, \S 12$ et $6, \S 3$, ainsi que considérant 19 du règlement « sécurité alimentaire » ;

${ }^{26}$ European Commission, op. cit., p. 39.

${ }^{27}$ Règlement CE n ${ }^{\circ}$ 178/2002, art. 3 13).
} 
est présentée comme un échange interactif entre tous les opérateurs du secteur alimentaire, y compris l'administration. La communication n'est pas envisagée à sens unique, mais avec des allers-retours, et ce tout au long du processus d'analyse des risques. Dès lors, la communication sur les risques constitue le ciment qui assure la stabilité et le fonctionnement de l'analyse des risques sanitaires des aliments.

Il s'agit donc de faire circuler entre les opérateurs des informations et des avis, pas seulement sur les risques, mais également sur les dangers, les facteurs liés aux risques et la perception de ceux-ci. Le législateur est resté plus discret quant à la définition de ces deux dernières notions, il est sans doute possible d'y voir une référence aux autres facteurs légitimes ${ }^{28}$. De manière générale, selon l'article 2 du règlement $(\mathrm{CE}) \mathrm{n}^{\circ} 178 / 2002$, le commerce des denrées alimentaires se doit d'être équitable et le cas échéant tenir compte « de la protection de la santé et du bien-être des animaux, de la santé des plantes et de l'environnement ${ }^{29}$, ce qui est finalement très lié à la santé humaine. Ces facteurs ne sont pas précisés dans l'article relatif à l'analyse des risques. Toutefois quelques précisions sont apportées au dix-neuvième considérant, qui indique qu'il s'agit "notamment des facteurs sociétaux, économiques, traditionnels, éthiques et environnementaux, ainsi que la faisabilité des contrôles ${ }^{30}$. En outre, l'autorité sanitaire des aliments doit évaluer certains aspects de la protection des travailleurs ${ }^{31}$. Concernant la perception des risques, il s'agit d'aller au-delà de la simple communication des résultats de l'évaluation des risques et d'adapter celle-ci à la manière dont les consommateurs reçoivent et comprennent l'information sur la sécurité sanitaire. En effet, le vocabulaire scientifique utilisé par l'évaluateur du risque n'est pas forcément compréhensible par le consommateur final. Par exemple, concernant les caractéristiques nutritionnelles d'une denrée alimentaire, le fait de communiquer sur la faible teneur en sodium ou en sel ne doit pas conduire l'opérateur à faire croire au consommateur que les eaux en question soient pauvres en sodium ou en sel ou conviennent pour un régime pauvre en sodium lorsque la teneur totale en sodium, sous toutes ses formes chimiques présentes, est égale ou supérieure à la limite réglementaire. Dans ce cas, la Cour de justice a estimé que le principe de précaution justifiait l'adoption de mesures restrictives ${ }^{32}$.

Mais les pouvoirs publics ont aussi parfois des discours normatifs incohérents. Au-delà de la qualité de la norme juridique, c'est sur les contradictions au sein même de la parole de la puissance publique que l'on peut s'interroger. Que faut-il comprendre lorsque l'agence de santé publique française recommande d'éviter les produits contenant des additifs, alors même que leur usage est autorisé par la législation alimentaire sur la base d'évaluations scientifiques ?

L'usage des additifs est encadré par le législateur européen. Le règlement (CE) n ${ }^{\circ}$ 1333/2008 énonce les additifs dont la mise sur le marché est autorisée. Conformément à ce règlement, et plus généralement au principe de l'analyse des risques, les additifs ont été réévalués à la lumière des nouvelles connaissances scientifiques depuis 2016. Pourtant, en 2019, l'agence de santé publique française recommande «par précaution » d'éviter les aliments ultratransformés, c'està-dire ceux « qui contiennent de nombreux additifs ». L'agence précise que l'on «n'en connaît pas encore précisément l'impact sur la santé humaine $»^{33}$. L'idée d'une réduction des additifs

\footnotetext{
${ }^{28}$ Ceux-ci peuvent être définis comme des normes juridiques ou éthiques supérieures ressenties comme fondamentales par la collectivité

${ }^{29}$ Règlement CE n ${ }^{\circ}$ 178/2002, art. 5 § 1 « Objectifs généraux ».

${ }^{30}$ Règlement CE n ${ }^{\circ} 178 / 2002, \S 19$.

31 Règlement CE n 178/2002, §37; Conseil National de 1'Alimentation, L'articulation entre le droit agroalimentaire et le droit du travail, Avis $\mathrm{n}^{\circ}$ 60, 3 avril 2008.

${ }^{32}$ V. par ex.: CJUE, 17 déc. 2015, Aff. C-157/14, Neptune Distribution SNC contre Ministre de l'Économie et des Finances.

${ }^{33}$ Santé Publique France, Recommandations relatives à l'alimentation, à l'activité physique et à la sédentarité pour les adultes, 2019, p. 35 .
} 
émerge chez le législateur français et l'interdiction de l'utilisation du dioxyde de titane a été considérée comme un « préalable indispensable $»^{34}$.

Les gestionnaires du risque portent ici des discours opposés à propos des risques liés aux additifs. D'un côté, l'agence de santé publique française porte un discours généraliste déconseillant la consommation de l'ensemble des additifs (y compris des additifs que l'on peut trouver naturellement dans des produits bruts comme l'acide citrique). De l'autre, des autorités publiques dont les mesures se doivent d'être précises et parfois divergentes. Concernant la nécessité d'être précis, comme l'a interprété la Cour de justice ${ }^{35}$, le droit européen s'oppose à ce qu'une législation nationale interdise globalement la mise sur le marché d'une catégorie de produits en se fondant sur une analyse des risques qui ne concerne que certains de ces produits. Ainsi, la force du principe de l'analyse des risques fondée sur les connaissances scientifiques peut aussi devenir une faiblesse, lorsque la mesure de précaution est restrictive et se doit d'être précise $^{36}$. En effet, la suspension de la mise sur le marché pose souvent le problème de la substitution de substances parfois très utilisées comme le glyphosate.

Ces contradictions ne peuvent que nuire à la confiance du consommateur dans le système d'analyse des risques, et sans doute aussi plus généralement à la confiance des citoyens dans la règle de droit qui s'érode. Comment expliquer que l'on autorise la mise sur le marché de produits d'un côté et recommander de limiter leur consommation de l'autre ? Le phénomène n'est pas nouveau, puisqu'il s'observe pour d'autres produits ou substances comme l'alcool. Pour ce produit, le risque pour la santé est avéré et c'est sans doute pour des raisons historiques et culturelles que sa mise sur le marché est peu restreinte. Est-ce alors parce qu'il ne faut pas nuire au modèle économique dominant les systèmes alimentaires ou parce que les maladies non transmissibles liées à l'alimentation ne sont pas si problématiques ? Toujours est-il que des doutes sur les risques liés à des produits amènent parfois à les laisser sur le marché.

Ces contradictions brouillent la communication sur le risque et perturbent de manière générale le processus de l'analyse des risques. Cela peut mener à ralentir ou paralyser le processus de décision ${ }^{37}$ ce qui est susceptible de profiter aux exploitants du secteur alimentaire. Cette situation n'est pas le seul fait des pouvoirs publics, la sécurité sanitaire des denrées alimentaires concernant les risques incertains est aussi assurée par les entreprises du secteur alimentaire qui ont également un devoir prudentiel.

\section{Opacité des mesures de précaution adoptées par les exploitants}

Dans un avis sur le recours au principe de précaution, le Comité économique et social (UE) estime que seul l'État a vocation à appliquer ce principe, les entreprises n'ayant pas les moyens de «déterminer ce qu'est un risque de dommage grave et nuisible, une mesure effective et proportionnée, un coût effectivement acceptable $»^{38}$. Pourtant, dans le domaine alimentaire, les textes européens, et le règlement $\mathrm{CE} \mathrm{n}^{\circ} 178 / 2002$ en particulier, énoncent à plusieurs reprises

\footnotetext{
${ }^{34}$ Assemblée Nationale, «Rapport d'information déposé en application de l'article 145 du règlement, par la commission des affaires économiques, en conclusion des travaux d'une mission d'information sur les conclusions de la commission d'enquête sur l'alimentation industrielle (Mme Michèle Crouzet) », sur Assemblée nationale [en ligne].

${ }^{35}$ CJUE, 19 janv. 2017, Aff. C-282/15, Queisser Pharma GmbH \& Co. KG contre Bundesrepublik Deutschland.

${ }^{36}$ N. De Sadeleer, Sécurité alimentaire et précaution, Sécurité alimentaire. Nouveaux enjeux et perspectives, Bruylant, 2013, [consulté le 3 juin 2020].p. 313.

37 V. not les enquêtes : S. Foucart, La fabrique du mensonge, 2014; S. Horel, Intoxication, La Découverte, 2015.

${ }^{38}$ Avis du 12 juillet 2000, JOCE C 268/6 du 19 septembre 2000, $\$ 3.4$ et 3.5 .
} 
des obligations en lien avec la suspicion d'un risque ${ }^{39}$. À la différence des mesures de prévention des autorités publiques, il est plus difficile d'observer les approches de précaution développées par les exploitants (A). S'ils sont concernés par une obligation de sécurité qui s'étend aux risques suspectés, les faits montrent que cela pourrait être renforcé par davantage de transparence $(B)$.

\section{A. Les exploitants concernés par les approches de précaution}

Le vocabulaire employé par le législateur européen utilise le registre de la précaution à propos des obligations reposant sur les exploitants du secteur alimentaire. Ces derniers doivent ainsi mettre en œuvre des mesures de retrait et de rappel et informer les autorités publiques lorsqu'ils ont « des raisons de penser qu'une denrée alimentaire ne répond pas aux prescriptions relatives à la sécurité des denrées alimentaires ». Ils doivent également informer les autorités compétentes lorsqu'ils ont « des raisons de penser qu'une denrée alimentaire qu'il a mise sur le marché peut être préjudiciable à la santé humaine ${ }^{40}$. De nature privée, ces pratiques sont aussi plus discrètes et donc plus difficiles à observer que celles des pouvoirs publics et elles n'ont pas été interprétées par la jurisprudence.

En sortant des sources classiques du droit, nous pouvons observer les faits portés à la connaissance du public et émettre quelques hypothèses. Les activités de certains lanceurs d'alerte et les crises récentes présentent un matériau fertile à quelques interrogations.

À l'automne 2019, la campagne de pétition lancée en France et en Allemagne par l'association Foodwatch sur des laits infantiles contaminés donne l'occasion d'observer la mise en œuvre de démarches de précaution par des exploitants du secteur alimentaire. Des analyses de laits en poudre destinés aux nourrissons ont mis en évidence la présence d'hydrocarbures aromatiques d'huiles minérales ${ }^{41}$. Ces substances ont été évaluées comme potentiellement cancérogènes, génotoxiques et perturbatrices endocriniennes et l'ANSES recommande de limiter l'exposition des populations ${ }^{42}$. Suite à la publication de ces résultats, les réponses des exploitants concernés sont intéressantes. Ils assurent que les produits peuvent être consommés en toute sécuritée ${ }^{43}$, mais mettent aussi en évidence toute la difficulté de caractériser ces risques à l'heure actuelle ${ }^{44}$. Ces réponses laissent supposer qu'ils connaissent ce risque incertain et qu'ils considèrent le maîtriser. En juin 2020, l'association a saisi par voie d'avocat la DGCCRF $^{45}$ et les préfets de France. La première pour qu'elle transmette des tests officiels qu'elle aurait réalisés et les seconds pour suspendre la mise sur le marché des produits concernés et ordonner des retraits et des rappels. En juillet 2020, Danone a annoncé que le lait contaminé n'était plus commercialisé. Si l'histoire semble loin d'être terminée à l'heure où ces lignes sont écrites, il est néanmoins

\footnotetext{
${ }^{39}$ Les exploitants alimentaires ne sont pas les seuls concernés par l'anticipation des risques, v. not. : F. Garcia, «L'anticipation des entreprises : Les obligations de collaborer / les obligations d'alerter », in François Rousseau et Karine Foucher (dir.), La réponse du droit aux crises sanitaires, L’Harmatan, 2016.

${ }^{40}$ Règlement CE n 178/2002, art. 19.

${ }^{41}$ L'association avait déjà mis en débat cette problématique en 2015 . Une précédente campagne d'analyse avait été menée par l'association sur un panel plus large de denrées alimentaires. Les résultats avaient alors montré que sur 42 produits testés, $60 \%$ étaient contaminés par des huiles minérales.

${ }^{42}$ Anses, «Avis de l'Agence nationale de sécurité sanitaire de l'alimentation, de l'environnement et du travail relatif à la migration des composés d'huiles minérales dans les denrées alimentaires à partir des emballages en papiers et cartons recyclés », publié le 8 mars 2017.

${ }^{43}$ V. par exemple la réponse de Nestlé en date du 28 octobre 2019 : https://www.foodwatch.org/fileadmin/FR/Documents/20191105_MinOil_Babymilk_echanges_foodwatch_nestle.pdf

$44 \quad$ V. l'échange avec Danone : https://www.foodwatch.org/fileadmin/FR/Documents/20191105_MinOil_Babymilk_echanges_foodwatch_danone.pdf

${ }^{45}$ Direction générale de la concurrence, de la consommation et de la répression des fraudes.
} 
loisible d'en tirer quelques enseignements concernant la place réservée aux approches de précaution par les exploitants du secteur alimentaire.

Si les opérateurs apparaissent conscients et mobilisés s'agissant des risques suspectés, le respect des obligations pesant sur les exploitants en matière de suspicion de risque n'est pas discuté juridiquement, et notamment devant les prétoires. Les obligations qui pèsent sur les exploitants du secteur alimentaire dans la mise en œuvre de démarches de précaution ne peuvent être effectives que si leur non-respect est susceptible d'engager leur responsabilité. La législation alimentaire européenne n'en énonce pas précisément et renvoie aux droits civils et pénaux nationaux. Or, l'engagement de la responsabilité des exploitants s'avère compliqué par la nature même de l'incertitude liée au risque et par la difficulté de démontrer son existence, en particulier dans le domaine alimentaire ${ }^{46}$. L'interprétation du juge concernant les mesures de précaution mises en œuvre par les exploitants manque, car elle permettrait de mieux les cerner. C'est volontairement qu'un standard est ouvert à une multitude d'interprétations " y compris à des situations futures et imprévues, et ne peut être mis en œuvre que par l'autorité judiciaire $\gg^{47}$. Cette judiciarisation pourrait en particulier trouver sa source dans des actions individuelles ou collectives de consommateurs. L'association Foodwatch se tourne d'ailleurs vers l'administration, et potentiellement les juridictions administratives pour faire évoluer la situation, mais une action devant les juridictions pénales est aussi envisageable. Cela ne semble pas être actuellement la stratégie des lanceurs d'alerte dans le domaine de l'alimentation, mais le dynamisme du contentieux autour des questions climatiques, des pesticides ou des médicaments pourrait les inspirer.

\section{B. La transparence au support de l'obligation de sécurité ?}

Au regard du traitement réservé au risque avéré par certains opérateurs, il est possible de s'inquiéter. L'affaire Lactalis rappelle que des négligences vis-à-vis d'un risque avéré existent et que des mesures de prévention, pour des raisons économiques, ne sont pas mises en œuvre ${ }^{48}$. De ce point de vue, il apparaît alors légitime de s'interroger sur la diligence de leurs actions menées en matière de risque suspecté.

Le législateur français en a pris acte. La loi dite «EGALIM » du 30 octobre $2018^{49}$ apporte des précisions quant à la mise en œuvre du règlement $(\mathrm{CE}) \mathrm{n}^{\circ} 178 / 2002$. Ainsi, l'exploitant doit désormais informer les autorités compétentes, « lorsqu'il considère ou a des raisons de penser, au regard de tout résultat d'autocontrôle, qu'une denrée présente un risque pour la santé humaine ou animale $»^{50}$.

Cette pression législative est bienvenue pour renforcer le système de sécurité sanitaire que ce soit vis-à-vis du risque avéré ou du risque suspecté, quoiqu'extrême en faisant référence à « tout résultat $»^{51}$. Mais le législateur français aura beau préciser ou répéter ce qui existe déjà dans le droit européen, il n'est pas capable de régler l'imprévisibilité et la variété des situations rencontrées par les exploitants alimentaires. Ce n'est d'ailleurs pas l'objectif, comme cela a

\footnotetext{
${ }^{46}$ J.-P. Bugnicourt, J.-S. Borghetti, F. Collart Dutilleul, « Le droit civil de la responsabilité à l'épreuve du droit spécial de l'alimentation : Premières questions ». Recueil Dalloz, 2010, pp.1099.

${ }^{47}$ L. Boy Laurence, « La nature juridique du principe de précaution », Nature Sciences Sociétés, 7, 1999.

${ }^{48}$ Sénat, Rapport d'information de Mme S. Primas et M. A. Milon, fait au nom de la commission des affaires économiques et de la commission des affaires sociales $\mathrm{n}^{\circ} 403$ (2017-2018) - 5 avril 2018.

${ }^{49}$ Loi n $^{\circ} 2018-938$ du 30 octobre 2018 pour l'équilibre des relations commerciales dans le secteur agricole et alimentaire et une alimentation saine, durable et accessible à tous, JORF n ${ }^{\circ} 0253 \mathrm{du} 1$ novembre 2018.

${ }^{50}$ C. rur., art. L. 201-7.

${ }^{51}$ Disposition tempérée par les modalités d'application précisées par une note technique des ministères en charge de la sécurité sanitaire des denrées alimentaires (Instruction technique DGAL/SDSSA/2019-555, juillet 2019)
} 
déjà été souligné. En effet, le principe de précaution peut être vu comme procédant «d'une délégation du pouvoir normatif à l'administration et/ou au juge $»^{52}$.

Dans l'affaire de contamination aux huiles minérales, nous pouvons constater un manque de transparence et des failles dans la communication sur les risques. D'un côté, les exploitants s'en tiennent à expliquer que les produits sont conformes à la réglementation et restent évasifs sur les autocontrôles réalisés. De l'autre, la nécessité d'un recours gracieux envers les autorités administratives montre que le système de l'analyse des risques fonctionne encore de manière imparfaite.

Au-delà de l'appréciation de l'opportunité de l'absence de mesures prises par les exploitants ou les pouvoirs publics ${ }^{53}$, ce sont à nouveau des dysfonctionnements de la communication sur le risque qui posent problème. La systématisation de la publicité de celle-ci paraît opportune pour combler certaines de ces imperfections. Rendre publics certains échanges et certaines informations aurait le mérite de montrer que les protagonistes de la sécurité sanitaire des aliments sont conscients du risque et que, si les questions sanitaires ne sont pas résolues, elles sont au moins prises en compte. En somme, que les acteurs de la gestion des risques n'ont rien à cacher.

Le cadre législatif est d'ailleurs favorable. L'article 10 du règlement $(\mathrm{CE}) \mathrm{n}^{\circ}$ 178/2002 prévoit une information des citoyens à propos des risques suspectés et autorise les autorités administratives à adopter des mesures appropriées. Des dispositifs de publicisation de la gestion des risques existent. Prévu par la loi d'avenir pour l'agriculture, l'alimentation et la forêt de 2014, le dispositif «Alim'confiance » organise par exemple la publication des résultats des contrôles officiels en matière de sécurité sanitaire des aliments. Accessibles en ligne ou affichées dans les locaux de l'établissement, les informations sont rendues publiques pour une durée minimale d'un an ${ }^{54}$.

Le dispositif «Alim'confiance » pourrait ainsi être enrichi en s'appuyant sur l'obligation de collaboration des exploitants du secteur alimentaire. Une diffusion systématique des résultats d'autocontrôle n'est sans doute pas nécessaire et porterait probablement une atteinte disproportionnée au droit de propriété. Par contre, une diffusion régulière et synthétique des autocontrôles et partant de l'identification et de la gestion des risques incertains pourrait être justifiée par l'objectif de sécurité sanitaire.

Face aux difficultés de mise en œuvre de la responsabilité dans le domaine alimentaire et aux différentes crises, il est également possible d'imaginer une réponse graduée par rapport à des comportements d'exploitants non conformes à cette obligation de collaboration renforcée. Une publication des mises en demeure de mise en conformité restées sans effet pourrait être un instrument adéquat ${ }^{55}$. La perte d'image de marque est parfois plus efficace pour faire évoluer les comportements des exploitants que celle d'une, non moins hypothétique, sanction administrative ou pénale.

\section{Conclusion}

\footnotetext{
52 C.-A. Morand, «Pesée d'intérêts et décisions complexes », La pesée globale des intérêts, Helbing \& Lichtenhahn, 1998, p. 47.

${ }^{53}$ Cette appréciation relèverait des juridictions.

${ }^{54}$ C. rur., art. D. 231-3-8 et suivant.

${ }^{55}$ Un tel dispositif existe en matière d'obligations d'actions de réduction de la consommation d'énergie finale dans des bâtiments à usage tertiaire (C. constr et hab., art. R. 131-44). D'une autre manière, l'article L. 171-8 du Code de l'environnement prévoit que «L'autorité administrative compétente peut procéder à la publication de l'acte arrêtant ces sanctions [administratives], sur le site internet des services de l'Etat dans le département, pendant une durée comprise entre deux mois et cinq ans. Elle informe préalablement la personne sanctionnée de la mesure de publication envisagée, lors de la procédure contradictoire prévue à l'avant-dernier alinéa du présent II ».
} 
Le principe de précaution et celui de l'analyse des risques sont des principes cardinaux de la législation alimentaire européenne. Si le bilan global de cette dernière s'est révélé positif, le système n'est pas parfait. À l'étude des approches de précaution mises en œuvre par les différents acteurs du secteur alimentaire, deux points peuvent être relevés. Le discours normatif des personnes publiques s'agissant du risque incertain est parfois incohérent et mal réparti par rapport aux compétences normalement dévolues aux différents pouvoirs, législatif et exécutif en particulier. À ce propos, une évidence peut-être rappelée : celle de la séparation des pouvoirs, qui favorise l'action du pouvoir exécutif dans le domaine du risque incertain. Pour les exploitants du secteur alimentaire, s'ils sont formellement concernés par la gestion du risque incertain, le manque d'interprétation jurisprudentielle et leur caractère secret rendent plus difficile l'analyse de ces approches.

Ce n'est pas tant l'imprécision des formes que peuvent prendre les mesures de précaution qui apparaît problématique, mais plutôt l'imprécision des contours de la communication sur les risques et le manque de transparence dans la collaboration entre les exploitants et les autorités compétentes. Des voies d'amélioration sont aussi possibles pour pallier ces limites intrinsèques du système de l'analyse des risques. L'importance du rôle des lanceurs d'alerte apparaît ici essentielle pour la mise en débat du choix des mesures à adopter face au risque suspecté. Leur activisme judiciaire pourrait favoriser une meilleure appréhension du risque suspecté. Les obligations des exploitants pourraient être affinées afin d'améliorer la transparence de leurs activités en matière de gestion du risque suspecté.

Ces précisions ne devraient toutefois pas nuire à la dimension atemporelle du principe de précaution, celle-ci «est précieuse, car elle redonne au droit toute sa dimension subversive/combative $»^{56}$.

Bibliographie :

Anses, « Avis de l'Agence nationale de sécurité sanitaire de l'alimentation, de l'environnement et du travail relatif à la migration des composés d'huiles minérales dans les denrées alimentaires à partir des emballages en papiers et cartons recyclés », publié le 8 mars 2017, https://www.anses.fr/en/system/files/ESPA2015SA0070.pdf

Assemblée Nationale, "Rapport d'information déposé en application de l'article $145 \mathrm{du}$ règlement, par la commission des affaires économiques, en conclusion des travaux d'une mission d'information sur les conclusions de la commission d'enquête sur l'alimentation industrielle (Mme Michèle Crouzet)», sur Assemblée nationale, http://www.assembleenationale.fr/dyn/15/rapports/cion-eco/115b2657_rapport-information

J. Bombardier, «Fabrication et distribution d'un produit : de la mise sur le marché à la gestion des risques, quelles obligations et sanctions pour les professionnels ? », Revue de droit rural, n²468, déc. 2018, étude 24.

R.-M. Borges, « Le statut des nouvelles techniques de sélection des plantes : entre incohérences juridiques et démission politique », RD rur., $\mathrm{n}^{\circ} 471$, mars 2019 , ét. 8 .

L. Boy, «La nature juridique du principe de précaution», Nature Sciences Sociétés, 7, juillet 1999, $\mathrm{n}^{\mathrm{o}} 3$, p. 5-11.

J.-P. Bugnicourt, J.-S. Borghetti, F. Collart Dutilleul, «Le droit civil de la responsabilité à l'épreuve du droit spécial de l'alimentation : Premières questions », Recueil Dalloz, 2010, pp.1099.

\footnotetext{
${ }^{56}$ L. Boy Laurence, « La nature juridique du principe de précaution », Nature Sciences Sociétés, 7, 1999.
} 
F. Collart Dutilleul, « Le principe de précaution dans le règlement communautaire du 28 janvier 2002 », in A. Massart, Ed. Giuffre (dir.), Prodotti agricoli e sicurezza alimentare, 2003.

Commission européenne, «The Regulatory Fitness and Performance Programme Evaluation of the General Food Law (Regulation (EC) No 178/2002), SWD(2018) 38 final », publié le 15 janvier 2018,

[https://ec.europa.eu/food/sites/food/files/gfl_fitc_comm_staff_work_doc_2018_part1_en.pdf Conseil National de l'Alimentation, Gestion de crise et communication, Enseignements tirés de la crise de l'ESB, avis n ${ }^{\circ} 79,2017$.

N. De Sadeleer, Sécurité alimentaire et précaution, Sécurité alimentaire. Nouveaux enjeux et perspectives, Bruylant, 2013, p. 307-345, http://tradeven.cluster015.ovh.net/wordpress/wpcontent/uploads/2018/12/Se_curite_alimentaire.pdf

D. Demortain, «Une société (de l'analyse) du risque?», Natures Sciences Sociétés, 27, octobre 2019, no 4, p. 390-398.

S. Foucart, La fabrique du mensonge: comment les industriels manipulent la science et nous mettent en danger, Gallimard., Paris, France, 2014, 409 p.

S. Horel, Intoxication: perturbateurs endocriniens, lobbyistes, eurocrates, Paris, France, La Découverte, 2015, 302 p.

S. Lacour, « Nanotechnologies. «Nanomatériaux dans l'alimentation. Vous reprendrez bien un peu de dioxyde de titane ?» », Cahiers Droit, Sciences \& Technologies, mars 2019, n 8, p. 213 $-224$.

C.-A. Morand, «Pesée d'intérêts et décisions complexes », La pesée globale des intérêts, Helbing \& Lichtenhahn, 1998.

F. Rousseau et K. Foucher (dir.), La réponse du droit aux crises sanitaires, L'Harmatan, 2016. Santé Publique France, Recommandations relatives à l'alimentation, à l'activité physique et à la sédentarité pour les adultes, 2019, p. 35.

Sénat, Rapport d'information de Mme S. Primas et M. A. Milon, fait au nom de la commission des affaires économiques et de la commission des affaires sociales $n^{\circ} 403$ (2017-2018) - 5 avril 2018. 